\title{
La congelación de las líneas de base y de los límites marítimos de los Estados insulares en riesgo de perder la totalidad de sus respectivos territorios debido al aumento en el nivel del mar ocasionado por el cambio climático
}

\author{
Alejandro José Velásquez Barrionuevo*
}

\begin{abstract}
RESUMEN
El presente artículo presenta la problemática actual del hundimiento de los espacios terrestres de determinados Estados insulares debido al aumento en el nivel del mar ocasionado por el cambio climático. Dicha problemática motivó la aprobación del tema «La elevación del nivel del mar en relación con el derecho internacional», en el programa de trabajo de la Comisión de Derecho Internacional de las Naciones Unidas, en particular respecto al ámbito de la condición de Estado. Se analizan las implicancias jurídicas del hundimiento total del espacio terrestre conforme al derecho internacional actual verificando que la final es la pérdida de la condición de Estado. A fin de dar solución a la problemática jurídica planteada, se propone la aplicación de una ficción jurídica por la cual se consideren congeladas las líneas de base de los Estados en riesgo, la cual ya fue presentada como una cuestión a estudiar en el ámbito antes mencionado por la Comisión de Derecho Internacional.
\end{abstract}

Palabras clave: cambio climático, carácter ambulatorio, Comisión de Derecho Internacional, condición de Estado, congelación, derecho del mar, derecho internacional, Estado ribereño, ficción jurídica, líneas de base, nivel del mar, territorio.

\footnotetext{
* Abogado con segunda especialidad en Derecho Internacional Público por la Pontificia Universidad Católica del Perú, es alumno de la Maestría en Derecho Internacional Económico y adjunto del curso Derecho Internacional Público de la misma universidad. Actualmente labora en el área internacional de la Dirección de Seguimiento y Evaluación del Ministerio de la Producción del Perú. Correo electrónico: avelasquezb@pucp.edu.pe
}

(iD) https://orcid.org/0002-2435-2225 
The freezing of baselines and maritime boundaries of insular States a t risk of losing all of their territories due to the rise in sea level caused by climate change

\section{Abstract}

This article presents the current problem of the sinking of the terrestrial spaces of certain insular states due to the rise in sea level caused by climate change. This problem led to the approval of the topic Sea-level rise in relation to international law in the programme of work of the United Nations International Law Commission, particularly in the area of statehood. The legal implications of the total sinking of the terrestrial space are analyzed in accordance with current international law, verifying that the final one is the loss of statehood. In order to solve the legal problem raised, the application of a legal fiction is proposed by which the baselines of the States at risk are considered frozen, which has already been presented as an issue to be studied in the aforementioned area by the International Law Commission.

Keywords: climate change, ambulatory nature, International Law Commission, statehood, freezing, law of the sea, international law, coastal state, legal fiction, baselines, sea level, territory.

\section{Introducción}

La presente investigación surgió en el marco de un tema de preocupación actual para la comunidad internacional, y se ha desarrollado con el objetivo de contribuir con argumentos jurídicos a la discusión sobre el tratamiento que el derecho internacional debe dar al territorio de los Estados que, por el aumento en el nivel del mar producido por el cambio climático, se encuentra actualmente reduciéndose y podría perderse (el espacio terrestre) en los próximos años.

Se propone reflexionar sobre la situación de los Estados que se encuentran actualmente bajo el riesgo de desaparecer debido al aumento en el nivel del mar producido por el cambio climático. El problema jurídico es cuál debe ser la respuesta del derecho internacional frente a la disminución y eventualmente la pérdida del espacio terrestre de los Estados por el aumento en el nivel del mar a causa del cambio climático a fin de que no se vean afectadas las zonas marítimas y, por ello, el territorio de dichos Estados.

Nuestra hipótesis es que la respuesta que debe dar el derecho internacional ante la disminución y pérdida del espacio terrestre de los Estados en riesgo de desaparecer por el aumento en el nivel del mar a causa del cambio climático es la creación de una ficción jurídica que implique que las líneas de base de dichos Estados se «congelarían» a fin de mantenerlas jurídicamente luego de la pérdida del espacio terrestre, así como sus límites marítimos. Para demostrar esto procedemos a revisar la normativa internacional y la doctrina existente en la materia. 


\section{Problemática actual de los Estados insulares en riesgo de perder su territorio a causa del aumento en el nivel del mar producido por el cambio climático}

El cambio climático ha traído consigo un incremento formidable en el nivel del mar en los últimos años. Cazenave y Remy mencionan que el último aumento considerable del nivel del mar fue el de 130 metros como producto de la desglaciación luego del último máximo glacial hace 21000 ańos y que luego de ello el nivel del mar se mantuvo relativamente constante. No es sino hasta fines del siglo XVIII e inicios del XIX que se evidencia un aumento de 0,7 milímetros anual coincidente con el inicio de la era industrial y el aumento se mantuvo en el siglo XX. Sin embargo, el mayor pico se encuentra en la década de 1990, ya que se detecta un aumento anual de 3,3 milímetros con margen de error de 0,4 milímetros desde 1993 a 2011. Las causas principales de este incremento considerable en los últimos años son la expansión termal del océano dado su calentamiento y el derretimiento de mantos de hielo y glaciares, y se calcula que el aumento va a continuar hacia lo que resta del siglo XXI (2011, pp. 648-651, 656).

Cifras muy cercanas son posteriormente presentadas por Ezcurra y Rivera-Collazo, quienes realizan un estudio enfocado en la elevación del mar en Puerto Rico, la cual es consistente con la tendencia mundial; al respecto, comentan que, desde aproximadamente el año 1900, la elevación del mar fue de 1,7 milímetros anual, mientras que, a partir de 1992, se calcula en 3,2 milímetros (2018, p. 3). Asimismo, hacia el otro lado del mundo, Siegel menciona que Yakarta, la capital de Indonesia situada en la isla de Java, se ha hundido de 3 a 10 centímetros por año desde 1974 a 2010, y que, en 2020, la tasa de hundimiento de dicha ciudad es calculada entre 20 a 25 centímetros anual $(2020$, p. 2). Teniendo en consideración estas cifras, que son de las más actualizadas disponibles, podemos concluir que, en efecto, el aumento en el nivel del mar continúa siendo actualmente un problema para distintos territorios en distintas partes del mundo, dado que se encuentran en un constante hundimiento que no solo no se detiene, sino que se mantiene en constante aumento con el pasar de los ańos.

Dado este escenario, que aparentemente no va a poder ser detenido de manera natural, se generan nuevos desafíos para el derecho internacional en diversas materias. Así, por ejemplo, Dremliuga reconoce que el derretimiento de los mantos de hielo y glaciares mencionado puede generar un problema con respecto a la interpretación del artículo 234 de la Convención de las Naciones Unidas sobre el Derecho del Mar de $1982^{1}$ (en adelante, la CONVEMAR), que regula el derecho de los Estados

\footnotetext{
1 La primera oración del artículo mencionado dice a la letra lo siguiente en su versión en español: «Los Estados ribereños tienen derecho a dictar y hacer cumplir leyes y reglamentos no discriminatorios para prevenir, reducir y controlar la contaminación del medio marino causada por buques en las zonas cubiertas de hielo dentro de los límites
} 
ribereños de dictar normas internas para proteger las zonas cubiertas de hielo de la contaminación de los buques que transiten por estas si se encuentran en sus zonas económicas exclusivas $(2017$, p. 2). La disposición mencionada exige que las zonas cubiertas de hielo se mantengan en dicha condición por más de seis meses al año, lo cual se dificulta en algunas zonas del ártico debido al derretimiento actual del hielo, y los Estados negociadores de la CONVEMAR no tomaron en cuenta el posible derretimiento futuro del hielo al crear esta norma (Dremliuga, 2017, pp. 2-3).

Uno de los problemas de mayor relevancia para el derecho internacional debido al aumento en el nivel del mar es que un grupo de Estados insulares de muy baja altura se encuentra en constante hundimiento de su espacio terrestre, lo cual puede llevar a que ese sea definitivo dada dicha altura. Al respecto, aproximadamente unos 40 Estados insulares se encuentran en dicho riesgo, aunque no existe una lista definitiva (Care, CIESIN, ACNUR, UNU-EHS y el Banco Mundial, citados por Park, 2011, p. 5). Como veremos más adelante, la mayoría de estos Estados aparentemente se encuentra en Oceanía. En todo caso, un problema anterior al hundimiento definitivo sería que las islas se vuelvan inhóspitas por factores meteorológicos, falta de agua dulce y aumento de enfermedades, todos ellos causados por el cambio climático (Park, 2011, p. 5).

En cualquiera de estos supuestos, siguiendo la línea más tradicional del derecho internacional, podríamos concluir que dichos Estados habrían perdido su territorio, lo cual implicaría que se haya perdido un elemento constitutivo del Estado y, por tanto, se pierda la condición de tal. Mencionamos esto dado que existen nuevas aproximaciones doctrinarias que buscan sustentar posibilidades distintas sobre la base del mismo derecho internacional actualmente existente; por ejemplo, Grote Stoutenburg menciona que la pérdida de la condición de Estado a causa del cambio climático podría violar normas imperativas como el derecho a la libre determinación y la soberanía permanente sobre los recursos y, por tanto, no se debería considerar tal (2013, p. 59).

A este punto, solo queremos hacer la precisión de que la terminología "condición de Estado» es utilizada en los informes oficiales en español de la Comisión de Derecho Internacional de las Naciones Unidas (en adelante, la CDI) (CDI, 2018a, p. 357, y 2019a, p. 374) como el equivalente al término Statehood de los informes en inglés (CDI, 2018b, p. 326, y 2019b, p. 340). Personalmente, creemos que la traducción no es la más exacta en nuestro idioma, en tanto no consideramos que ser Estado

\footnotetext{
de la zona económica exclusiva, donde la especial severidad de las condiciones climáticas y la presencia de hielo sobre esas zonas durante la mayor parte del año creen obstrucciones o peligros excepcionales para la navegación, y la contaminación del medio marino pueda causar daños de importancia al equilibrio ecológico o alterarlo en forma irreversible». Según Dremliuga, dicha disposición forma parte de la costumbre internacional en la materia (2017, p. 2).
} 
sea una "condición» como tal y habríamos preferido que se utilizara «estatalidad», traducción más literal referida por la doctrina en español, como López Martín, por ejemplo (2018, pp. 105-106). Sin embargo, la presente investigación se basa en gran parte en el trabajo de la CDI, la cual, al ser un órgano de la ONU, da valor a sus textos oficiales en idioma espańol, por lo que, para efectos del presente artículo, se utilizará dicha terminología al hacer referencia al tipo de subjetividad internacional con la que cuentan los Estados en aras de ser fiel al léxico utilizado por la CDI en idioma español.

El problema en relación a la condición de Estado parte de la definición misma de dicho sujeto de derecho internacional, la cual, independientemente de la teoría doctrinaria que se siga, incorpora necesariamente como uno de sus elementos al territorio, el cual puede ser definido de manera simplificada como aquel espacio geográfico en el que ejerce su soberanía (Crawford, 2006, p. 55). El espacio terrestre de un Estado insular podría verse eventualmente cubierto en su totalidad por el agua del mar como producto del cambio climático, lo cual implicaría, conforme a Blanchard, que ya no exista más, en tanto no puede haber una comunidad organizada bajo el agua (2015, p. 78). Asimismo, siguiendo a Grote Stoutenburg (2013, p. 60), dado que el territorio no es un fin en sí mismo, sino la base física en que la población vive en comunidades organizadas, incluso se entendería que el Estado ha perdido su territorio antes de que este se hunda totalmente: en el momento en el que se vuelve inhabitable, dado que ya no es capaz de albergar a su población. No obstante, esta afirmación debe ser entendida solo en el contexto en el que la totalidad del territorio de un Estado queda inhabitable, ya que el hecho de que uno de varios espacios terrestres sea inhabitable no desnaturaliza que sea parte del territorio del Estado, como se ve en el caso de las rocas en el derecho del mar (ONU, 1982, art. 121.3).

Entre los casos de mayor riesgo, Park reconoce que existen Estados como Maldivas, Tuvalu e Islas Marshall, que cuentan con su punto más alto a menos de diez metros sobre el nivel del mar, los que corren el riesgo más grave ya que podrían quedar hundidos por completo con mayor rapidez que los demás; así, por ejemplo, se calcula que Tuvalu podría desaparecer hacia la segunda mitad del presente siglo (2011, p. 4). No obstante, existen más casos de Estados en riesgo, si bien no de sumergirse por completo con tanta prontitud, sí de quedar inhabitables como Kiribati, que actualmente se encuentra en proceso de búsqueda de opciones para la reubicación de su población, lo cual pretende lograr con medidas como la cesión en su favor de territorios de terceros Estados (McAdam, 2010, p. 122) o la compra de instalaciones flotantes artificiales (Grote Stoutenburg, 2013, p. 63). 
El Estado que actualmente parecería generar mayor preocupación es Maldivas, que ha realizado varios actos a fin de salvaguardar a su población, así como su espacio terrestre. Entre ellos, Maldivas planteó la compra de territorios a India y Australia a fin de reubicar a su población (McAdam, 2010, p. 122) y construyó una isla artificial denominada Hulhumalé de 188 hectáreas en la cual inició la reubicación de su población en 2004 (Grote Stoutenburg, 2015, p. 169). Actualmente, esta última representa el punto más alto sobre el nivel del mar en Maldivas con 2,1 metros, por encima de los 1,5 metros que cuentan sus islas naturales como punto más alto (Misión Permanente de la República de Maldivas en las Naciones Unidas, 2019, p. 8). Una de las islas mencionadas es precisamente la que dio el nombre a la isla artificial y que es además la capital del Estado, denominada Malé (República de Maldivas, 2008, Art. 14). Comparando la información señalada con otra fuente de información sobre la altura de Maldivas, la cual fue proporcionada por la Agencia Central de Inteligencia de los Estados Unidos, se identificó que la altura máxima de Maldivas en el año 2008 fue de 2,4 metros (citada por Park, 2011, p. 4). Siendo así, se puede identificar que, si bien es gradual, el hundimiento es también cada vez más rápido.

Otro grupo de Estados particularmente afectado y en riesgo por el aumento del nivel del mar está integrado por los miembros del Foro de las Islas del Pacífico (PIF por sus siglas en inglés): los Estados Federados de Micronesia, Fiji, Nauru, Palau, Papúa Nueva Guinea, Samoa, Islas Salomón, Tonga y Vanuatu, además de Kiribati, Islas Marshall y Tuvalu, mencionados anteriormente; también serían afectadas Australia y Nueva Zelanda, aunque estos últimos se encuentran en un riesgo evidentemente menor debido a sus extensiones territoriales mayores y con mayor altitud sobre el nivel del mar. A finales de 2019, el PIF declaró ante la Organización de las Naciones Unidas (ONU) que el océano Pacífico se encuentra particularmente afectado por el aumento en el nivel del mar y esto amenaza el territorio de sus miembros por afectar el espacio terrestre y la extensión de las costas de sus miembros, el desarrollo sostenible, el sustento de vida de los mismos, y la nacionalidad de sus nacionales (Foro de Islas del Pacífico con Misiones en las Naciones Unidas, 2019, p. 1).

No obstante, no toda la problemática se resume a la posibilidad de pérdida total del espacio terrestre de determinados Estados insulares con baja altura sobre el nivel del mar, sino que también el hundimiento parcial del territorio de las costas de los Estados ribereños que ven reducido su territorio costero debido al aumento en el nivel del mar. Como mencionamos anteriormente, Australia y Nueva Zelanda plantean que este es un problema que comparten con los demás miembros del Foro de las Islas del Pacífico y, a ellos, se pueden sumar los ejemplos de Kotzebue Sound en Alaska, que ha visto reducida su entrada de 23,9 a 25,8 millas marinas (lo que supuso un reajuste obligatorio de las líneas de base de Estados Unidos en este punto) o las 
costas frente a frente de Cuba y Estados Unidos en Florida que cuentan con cayos de altura baja que se podrían hundir y que podrían afectar inclusive a terceros Estados como Bahamas (Aznar Gómez, 2013, p. 36).

Debido a toda la problemática identificada anteriormente, la Comisión de Derecho Internacional (CDI) de las Naciones Unidas aprobó la inclusión del tema «La elevación del nivel del mar en relación con el derecho internacional» como parte de su programa de trabajo en el año 2019, planteando como ámbitos a estudiar los relativos al derecho del mar, la protección de las personas afectadas por la elevación del nivel del mar y la condición de Estado (CDI, 2019a, p. 374). La CDI se encuentra estudiando las posibles respuestas por parte del derecho internacional a la problemática en tanto se pueden afectar las zonas marítimas, los derechos de los nacionales y la condición de Estado de los Estados que se encuentran afectados por el aumento en el nivel del mar. No obstante, al día de hoy, solo se ha presentado un avance en relación a este trabajo.

En el siguiente punto, pretenderemos brindar las posibles soluciones a esta problemática desde el derecho internacional actual. Al respecto, mencionaremos las características de las líneas de base de los Estados ribereños, la implicancia que tienen en relación al territorio de dichos Estados, y la posible pérdida de la condición de Estado a causa de la pérdida del espacio terrestre. Finalmente, luego de presentar el panorama general que ofrece el derecho internacional actual, propondremos una nueva solución, la cual ya ha sido presentada por la CDI como la tercera cuestión a estudiar del ámbito de la condición de Estado de su tema «La elevación del nivel del mar en relación con el derecho internacional», antes mencionada, la cual es la «congelación» de las líneas de base de los Estados afectados a fin de mantener sus condiciones de Estado (CDI, 2018a, p. 360).

\section{Respuestas a la problemática territorial de los Estados insulares a partir del derecho internacional actual}

Este punto presentará el panorama actual del derecho internacional en relación con los asuntos de relevancia para la problemática identificada en el punto 2: el carácter que tienen las líneas de base en el derecho del mar, la conexión de las zonas marítimas con el espacio terrestre y la "condición de Estado» (en los términos de la Comisión de Derecho Internacional) frente a la eventual pérdida del territorio en sus ámbitos terrestre, marítimo y aéreo. 


\subsection{El carácter «ambulatorio» de las líneas de base en el derecho del mar}

La idea en este subpunto es mostrar el llamado carácter «ambulatorio» de las líneas de base en el derecho del mar que implica que deben actualizarse conforme a los cambios geográficos que se susciten de manera natural a través de las cartografías oficiales de los Estados ribereños. Para estos efectos, explicaremos qué son las líneas de base y cómo se las establecen los Estados ribereños, estableceremos por qué los cambios geográficos dan lugar a una actualización de las líneas de base, determinaremos cómo se actualizan las líneas de base, y precisaremos por qué se considera que las líneas de base tienen un carácter ambulatorio.

Caron (1990, p. 631) identifica que el surgimiento de la figura de las líneas de base resulta coincidente con el inicio del establecimiento de la anchura en el mar territorial, primera zona marítima reconocida como reivindicable por los Estados ribereños; asimismo, el autor menciona que el primer momento en el cual se plantea una anchura determinada fue con la carta de Thomas Jefferson como secretario de Estado de Estados Unidos a Francia y Gran Bretaña en 1793 y que en el siglo XIX se empezó a expandir la idea de que debía existir una anchura que se mediría desde una marca de agua que empezó a ser denominada «línea de base», lo cual terminó por ser extendido considerablemente para el siglo XX. Así, los primeros intentos de regulación de la institución se dieron en el seno de la Liga de las Naciones, en la Conferencia de Estados en La Haya de 1930, en la cual se impuso la postura que permitiría posteriormente regular las líneas de base para poder expandir las zonas marítimas a partir de ellas (Caron, 2009, p. 4). Siguiendo todo lo señalado, podemos definir de manera personal a las líneas de base como la marca de agua a ser trazada de forma unilateral por los Estados ribereños para medir la anchura de sus zonas marítimas.

Se pueden encontrar diversas disposiciones en que se hace referencia a las líneas de base en la CONVEMAR. En cuanto al trazado de las mismas, vamos a encontrar una regla general y algunas reglas especiales. Respecto a la primera, el artículo 5 de la CONVEMAR establece que, para medir la anchura del mar territorial, como regla general, se trazará la llamada línea de base «normal» y establece que será «la línea de bajamar a lo largo de la costa, tal como aparece marcada mediante el signo apropiado en cartas a gran escala reconocidas oficialmente por el Estado ribereño" (ONU, 1982). El antecedente directo de esta disposición es el artículo 3 de la Convención de Ginebra de las Naciones Unidas sobre el Mar Territorial y la Zona Contigua de 1958, adoptada tras la primera conferencia de la ONU sobre derecho del mar, la cual cuenta con una estructura similar (ONU, 1958). De estas disposiciones, se pueden extraer distintas definiciones para las líneas de base, como la presente en el Diccionario de Derecho de Black, la cual es la línea que divide la tierra del mar, mediante la cual se mide la extensión de la jurisdicción costera de un Estado (Garner, 
2004, p. 453). Con esto último, debemos entender que se hace referencia general a la anchura de las zonas marítimas y no solo del mar territorial.

No obstante, esta es solo una de las formas con las cuales los Estados pueden establecer sus líneas de base, ya que se reconocen hasta tres adicionales: líneas de base rectas, líneas de base archipelágicas y líneas de base de cierre a través de desembocaduras de ríos y bahías (Tanaka, 2019, p. 54). Todas ellas pueden resultar relevantes para efectos de los Estados insulares en riesgo de desaparecer y en general de los Estados ribereños que podrían ver afectadas sus costas, ya que la posibilidad de aplicarlas depende de condiciones geográficas específicas. Las primeras se encuentran reguladas en los artículos 7 y 10 de la CONVEMAR, los cuales tienen como antecedentes directos los artículos 4 y 7 de la Convención de Ginebra previamente mencionada. Las disposiciones mencionadas establecen diversos criterios para que se permita a los Estados ribereños, básicamente por accidentes geográficos que existan a su favor en sus costas, que puedan cerrar estos accidentes en líneas de base conformadas por líneas rectas, lo cual permitirá que el agua del mar que se encuentre dentro de dichas líneas de base califique como aguas interiores. Así, podemos entender que este tipo de línea de base es la de la distancia más corta entre dos puntos que se derivan de acuerdo con la CONVEMAR (Walker, 2012, p. 306).

Con relación al segundo tipo, hay casos en que un Estado insular puede contar con los requisitos previstos en el artículo 47 de la CONVEMAR para conformar un archipiélago que permita trazar líneas de base archipelágicas, las cuales cuentan con beneficios mucho mayores a los de las líneas de base normales, ya que permitirá aplicar un tipo especial de líneas de base rectas, que también significarán un tipo de línea de base de la distancia más corta entre dos puntos derivados de la CONVEMAR (Walker, 2012, p. 306). Los criterios son netamente técnicos y se encuentran en 10 numerales del artículo 47 ya señalado, por lo que se deben cumplir con las medidas geográficas y matemáticas exigidas para poder válidamente trazar este tipo de línea de base. Ocurre lo propio con el último tipo, regulado en los artículos 9 y 10 de la CONVEMAR, en tanto cuenta con determinados criterios técnicos dependientes de la existencia de accidentes geográficos como son las desembocaduras de ríos y bahías hacia el mar territorial del Estado ribereño.

En ese sentido, los Estados ribereños pueden optar por una serie de criterios distintos para efectos de trazar sus propias líneas de base, en tanto la determinación de qué criterio se utilizará para estos efectos y el propio acto de trazarlas los realizará el propio Estado ribereño, lo cual tiene como fundamento final la igualdad soberana de los Estados. Esto además fue establecido desde muy temprano por la propia Corte Internacional de Justicia en el caso Pesquerías del Reino Unido contra Noruega (1951, p. 152). Sin embargo, es importante tener en consideración que, como menciona 
Tanaka, el carácter unilateral de estos actos no implica que pueda no cumplirse con los requisitos establecidos por el derecho del mar para trazar uno u otro tipo de línea de base, ya que, de no cumplir con los criterios establecidos, las líneas de base no tendrán valor en relación a los demás Estados que se puedan oponer (2019, p. 61). Es importante tener en consideración entonces que este carácter unilateral apunta a que la soberanía sea ejercida en el marco del derecho del mar, por lo que jurídicamente se tiene ese límite. Como veremos a continuación, el cumplimiento de los criterios se encuentra sujeto a posibles cambios geográficos en las costas de los Estados ribereños.

Sucede que, en aplicación del artículo 76.9 de la CONVEMAR, los Estados partes de dicho tratado podrían comunicar al Secretario General de la ONU la anchura de la plataforma continental sobre la cual ejerce jurisdicción. En caso de ocurrir esto, el límite exterior de la plataforma continental se considerará inalterado en el tiempo hasta que haya una nueva declaración por parte del Estado ribereño. Del contenido de esta disposición, se puede concluir que la jurisdicción que ejerce el Estado ribereño sobre su plataforma continental no cuenta de por sí con carácter permanente, sino que es necesario notificar su alcance al secretario general de la ONU para que adquiera jurídicamente tal carácter. Asimismo, dado que no existe una disposición similar para ninguna de las otras zonas marítimas y que todas ellas se miden a partir de las líneas de base del Estado ribereño, se puede concluir que las líneas de base (y, por consiguiente, las zonas marítimas distintas a la plataforma continental tras la comunicación al secretario general de la $\mathrm{ONU}$ ) cuentan con un carácter no permanente geográficamente hablando. En contraposición, las líneas de base cuentan con un carácter de no permanencia geográfica por regla general, lo que implica que se encuentran jurídicamente hablando en un constante movimiento. A este carácter, autores de la doctrina anglosajona como Caron o Powers y Stucko han decidido denominar ambulatory, haciendo referencia a que, por más que sean fijadas por el Estado ribereño, se encuentran sujetas al movimiento natural de la geografía del mundo que podría afectar eventualmente a sus costas. Así, Caron menciona que dicho carácter genera dos efectos, los cuales son que, si se sumerge un punto sobre el cual se traza la línea de base, esta se debe volver a trazar a partir de los puntos que aún existen, y que las zonas marítimas que se proyectaban desde la línea de base anterior, se deberán adaptar a la nueva línea de base $(2009$, p. 9), por lo que entiende que se hace extensible a las zonas marítimas distintas a la plataforma continental comunicada al Secretario General de la ONU (1990, p. 635). Por otro lado, Powers y Stucko mencionan que, si bien este carácter no está expreso en la CONVEMAR, se puede concluir de la misma y que la Corte Internacional de Justicia no lo ha tratado directamente, pero admite la flexibilidad en las líneas de base (2013, p. 130). 
Aznar Gómez hace referencia en nuestro idioma al carácter señalado y lo traduce de manera indistinta refiriéndose a líneas de base «ambulatorias» y fronteras «deambulatorias" (2013, pp. 37 y 39). Sin embargo, preferimos el primero de los términos por considerarlo más cercano al original. En ese sentido, queda claro que los autores antes mencionados, especialistas en derecho del mar, entienden que las líneas de base, y por consiguiente las zonas marítimas a excepción de la plataforma continental notificada al secretario general de la ONU, cuentan con un carácter «ambulatorio», que implicará la cancelación geográfica de las líneas de base cuando se dejen de cumplir los criterios establecidos por el derecho del mar para que estas existan en un punto geográfico determinado y su necesaria adaptación a los puntos que aún existan, lo que llevará consigo el movimiento necesario también de las zonas marítimas medidas a partir de dichas líneas de base.

La forma en que se deben realizar estas adaptaciones es nuevamente a través del propio Estado ribereño de manera unilateral, pero respetando los criterios del derecho del mar para estos efectos. Así, por ejemplo, si un Estado insular contaba con líneas de base archipelágicas válidamente porque cumplía con los criterios geográficos establecidos en el artículo 47 de la CONVEMAR, pero debido a un cambio en la geografía ahora ya no cuenta con el cumplimiento de alguno de los criterios, deberá adaptar sus líneas de base a lo que le sea posible en tanto ya no contará con los requisitos jurídicos para mantener sus líneas de base archipelágicas (Rayfuse, 2012, p. 151). Solo por ilustrar uno de estos criterios que podrían perderse por motivos geográficos, el numeral 2 del artículo 47 de la CONVEMAR establece que se debe cumplir con que las líneas de base no excedan 100 millas marinas salvo hasta un 3\% del número total de líneas de base que encierren un archipiélago hasta un máximo de 125 millas marinas. Debido a algún cambio geográfico, podrían ya no cumplirse estas medidas necesarias. Aparentemente, nada impediría que se utilicen otros criterios, pero deben ser conformes al derecho del mar.

Finalmente, la adaptación práctica de las líneas de base se realiza en función a lo previsto en el artículo 16 de la CONVEMAR, que establece que deben encontrarse en cartas y listas de coordenadas oficiales realizadas por el Estado ribereño y publicitadas a través de su depósito a través del Secretario General de la ONU. Symmons y Reed identifican que esta publicidad es relevante para efectos de que los marinos que se puedan encontrar navegando por las zonas marítimas de un Estado ribereño tengan conocimiento de cuál es el tipo de línea de base que ha trazado el Estado, dado que de ello dependerá hasta dónde llegarán sus zonas marítimas y, por tanto, las libertades que haya en ellas (2010, p. 80). A todo esto, agrega Tanaka que, en realidad, para efectos prácticos, no se realiza una actualización de las líneas de base conforme a los cambios geográficos en tiempo real, sino que los propios Estados realizan sus cambios 
con una discrecionalidad considerable (2019, p. 55). Esta discrecionalidad se traduce tanto en el contenido de los cambios como en el momento en que se van a realizar; esto último ya que los Estados ribereńos evalúan el momento propicio para realizar sus cambios y, si no les conviene realizarlos con celeridad, se podrán demorar en llevarlos a cabo en tanto no hay una obligación de hacerlo en un plazo determinado.

En conclusión, las líneas de base cuentan indiscutiblemente con un carácter ambulatorio, lo cual implica que se encuentren en constante movimiento y necesaria actualización por parte de los Estados ribereños. Esto también afecta por consiguiente a la extensión de las zonas marítimas que se miden desde las líneas de base, con la excepción de la plataforma continental cuando su anchura y límites han sido comunicados al secretario general de la ONU en virtud de la disposición del artículo 76.9 de la CONVEMAR, aplicable solo para Estados partes de dicho tratado.

\subsection{La pérdida del espacio terrestre y de la condición de Estado}

En este subpunto, veremos que el territorio es un elemento constitutivo del Estado. Asimismo, explicaremos qué ocurre con las zonas marítimas de un Estado ribereño que ha perdido la totalidad de su espacio terrestre. Finalmente, desarrollaremos cuál sería la consecuencia jurídica de perder el territorio en relación con la «condición de Estado» en los términos de la CDI.

En primer lugar, es importante señalar que no existe una doctrina única que señale de manera inequívoca cuáles son los elementos constitutivos de un Estado. Un primer intento de llegar a dicho consenso se dio en el ámbito americano a través de la Convención sobre Derechos y Deberes de los Estados de 1933, mejor conocida como el Tratado de Montevideo. Dicho tratado establece en su artículo 1 que son elementos del Estado los siguientes: 1. Población permanente, 2. Territorio determinado, 3. Gobierno y 4. Capacidad de entrar en relaciones con los demás Estados (1933, Art. 1). Así, podemos ver que un elemento es, según el numeral, un territorio determinado. Sin embargo, dicho tratado se dio en un ámbito regional y no tendría aplicación universal.

Es así como existen posturas doctrinarias que varían los elementos previstos en dicha norma, tales como la de Crawford, quien seńala que uno de los elementos es la «independencia» (2012, p. 130), en lugar del elemento del numeral 4 llamado "capacidad de entrar en relaciones con los demás Estados». Sin embargo, resulta ser que el territorio siempre es un elemento presente en la doctrina. Entre otros autores fuera del continente americano que consideran al territorio como un elemento constitutivo del Estado independientemente de los demás elementos, encontramos a Remiro Brotóns et al. (2010, p. 69); Aust (2005, p. 16); Diez de Velasco (2013, p. 280); y 
Shaw, quien además agrega que existe una necesidad de que el Estado cuente con una base territorial definida en la cual pueda operar, lo que no implica necesariamente que esta se encuentre delimitada (2006, p. 199). Por otro lado, no hay ninguna norma internacional de carácter consuetudinario general que determine cuáles son los elementos del Estado.

En segundo lugar, el derecho del mar puede ser entendido como un régimen especializado del derecho internacional en los términos de la CDI (2006, párrafos 135 y 152 , entre otros), en tanto se trata de un sistema que, si bien se encuentra igual sujeto al derecho internacional general, cuenta con sus propias normas y principios especiales (la gran mayoría de ellos codificados en la CONVEMAR y también presentes en la costumbre internacional). En general, el hecho de que los requisitos jurídicos para trazar los distintos tipos de líneas de base sean geográficos en relación con la formación de las costas responde a un principio propio del derecho del mar: «la tierra domina el mar» (Aznar Gómez, 2013, p. 43).

Este principio implica que no es posible que un Estado cuente con zonas marítimas si es que no cuenta con un espacio terrestre desde el cual proyectarlas. Así, por ejemplo, se evita que Estados que no son ribereños puedan ejercer derechos de soberanía o incluso soberanía plena y exclusiva sobre espacios marítimos en tanto no cuentan con un espacio terrestre con acceso físico al mar como para poder proyectar zonas marítimas en dichos espacios. La Corte Internacional de Justicia reconoció este carácter de las zonas marítimas desde temprano en su actividad jurisdiccional en el caso Pesquerías previamente citado (1951, p. 133).

En ese sentido, el hecho de que un Estado eventualmente perdiera su territorio, como sería el caso de los Estados insulares en riesgo de los cuales ya hablamos en el punto anterior, implicaría, siguiendo el principio señalado del derecho del mar, que se pierdan también sus zonas marítimas. Así, no sería posible, por ejemplo, alegar válidamente que el Estado, si bien ha perdido su espacio terrestre, no ha perdido su territorio marítimo, en tanto este último necesita del primero para poder existir. Es importante resaltar que ocurre algo similar con el espacio aéreo, ya que se trata de una columna vertical de aire que se proyecta por encima del espacio terrestre y marítimo (Fernández Huertas, 1984, p. 36). Siendo así, el espacio aéreo como territorio del Estado seguiría también una suerte de carácter complementario a otros espacios geográficos que conforman el territorio en tanto estaría relacionado a estos otros espacios a fin de determinar su punto de partida.

Uniendo las dos ideas que hemos esbozado hasta ahora, si un Estado insular perdiera por completo su espacio terrestre, ello conllevaría la pérdida de su mar territorial y de su espacio aéreo, por lo que se entendería que habría perdido la totalidad de su 
territorio. En ese sentido, teniendo en cuenta que el Estado habría perdido uno de sus elementos constitutivos, la consecuencia jurídica lógica sería que ha perdido su condición de Estado. Es cierto que se han dado propuestas por parte de la doctrina para interpretar que esta no sería la consecuencia jurídica para darse, tales como la del «Estado hundido» (McAdam, 2010, p. 106), el "Estado desterritorializado» (Crawford y Rayfuse, 2012, p. 250) o incluso el "Estado en el exilio» (Crawford y Rayfuse, 2012, p. 253). Sin embargo, estas son solo propuestas que no cuentan con aceptación general de la comunidad internacional.

En conclusión, al ser el territorio un elemento presente en todas las definiciones que se puedan dar en la doctrina sobre el Estado como sujeto de derecho internacional, este se entiende como indispensable para la continuación de la condición de Estado. Sin embargo, la eventual desaparición del espacio terrestre de un Estado insular no llevaría a interpretar que se mantiene su mar territorial (ni ninguna otra zona marítima) ni su espacio aéreo, sino, por el contrario, que se han perdido también como consecuencia de la pérdida del espacio terrestre. Esto implicaría una pérdida total del elemento territorio de la definición de Estado, lo cual implicaría que el Estado perdería su condición de tal.

\section{La ficción jurídica por la cual se plantea «congelar» las líneas de base de los Estados insulares en riesgo de perder su espacio terrestre por el cambio climático}

A diferencia del anterior, este punto pretende justificar la eventual aplicación de una ficción jurídica por la cual se entienda que el carácter «ambulatorio» de las líneas de base se cambie por un carácter estático, lo cual además tendrá como consecuencia que se mantengan los límites marítimos que ya existan previamente. El punto explicará inicialmente la posibilidad previamente identificada de aplicar ficciones jurídicas en el derecho internacional. Luego de ello, se presentará la propuesta de crear esta ficción jurídica a partir del informe de la septuagésima sesión de la CDI y se analizará en función a los comentarios de la doctrina. Finalmente, se pretenderá justificar la aplicación de dicha ficción a partir de los beneficios que podría traer la aplicación de la misma a los Estados en riesgo de desaparecer como también a los demás Estados ribereños del mundo.

\subsection{La posibilidad de aplicar ficciones jurídicas en el derecho internacional}

En este subpunto, se explicará brevemente la posibilidad de aplicar ficciones jurídicas en el derecho del mar y en el derecho internacional en general, y se analizarán las ventajas de su aplicación. 
El Diccionario de Derecho de Black, citado anteriormente, utiliza el vocablo en inglés legal fiction para hacer referencia a una suposición de que algo es cierto, inclusive cuando podría ser falso (Garner, 2004, p. 2617). La referencia a dicha suposición se da en el ámbito jurídico al tratarse de una ficción creada por el derecho. Partiendo de la base de esta definición, podemos encontrar múltiples ficciones jurídicas en el derecho internacional, es decir, presunciones que implican que se encuentre ya supuesto un resultado jurídico que puede ser distinto a los hechos de la realidad.

Así, por ejemplo, se sostiene que, ante la ausencia del elemento constitutivo del Estado relativo al Gobierno, en términos del Tratado de Montevideo (o el elemento que se corresponda con este dependiendo del enfoque doctrinario a seguir), se aplica un principio de continuidad del Estado, que implica que, por más que temporalmente el Estado haya perdido un elemento constitutivo, mantendrá su condición de tal (Crawford, 2006, p. 86). Es importante resaltar que este principio no sería aplicable en el caso del elemento territorio si su pérdida se tratara del sumergimiento debido al aumento en el nivel del mar, puesto que la pérdida sería definitiva en tanto no habría propiamente una forma de recuperar ese territorio (Grote Stoutenburg, 2015, p. 296). Siguiendo esto, si un Estado cae en un determinado vacío de poder transitoriamente, siguiendo esta doctrina, en el fondo lo que operará será una ficción jurídica que presumirá que el Estado sigue manteniendo sus elementos constitutivos, a pesar de que, en los hechos, no cuenta con todos.

Según la apreciación de otros autores, existirían más ficciones jurídicas en la asunción de ciertos hechos tales como que los Estados no puedan haber tenido la voluntad de haber creado normas que sean contradictorias entre sí (Milanovic, 2014, p. 109) o que el daño hecho por un Estado a un extranjero bajo su jurisdicción implique que el daño sea recibido por el Estado del cual el extranjero es nacional (Sornarajah, 2010, p. 121). Se ve entonces que la institución de la ficción jurídica se encuentra presente en el derecho internacional.

Además de las ficciones jurídicas relativas al derecho internacional general, el derecho del mar como un régimen especializado de este último, no es ajeno a la existencia de estas. La más relevante a efectos del presente artículo es aquella ya mencionada sobre la cual se desprende que las líneas de base cuentan con un carácter "ambulatorio", y es aquella establecida en el artículo 76.9 de la CONVEMAR, que establece que el límite exterior de la plataforma continental notificada al secretario general de la ONU por parte de los Estados partes de la CONVEMAR será permanente.

Por otro lado, autores como Strati (2006, p. 29) y Churchill y Lowe (1988, p. 117) reconocen que la CONVEMAR establece una ficción jurídica que puede ser deducida a partir de la lectura conjunta de sus artículos 33 y 303.2 que implica que los 
Estados ribereños mantengan su soberanía hasta un máximo de 24 millas marinas contadas desde las líneas de base en lo que respecta a la zona contigua en relación con objetos históricos y arqueológicos en ella. $\mathrm{Al}$ respecto, el Estado ribereńo tiene la facultad de reivindicar una zona marítima denominada zona contigua hasta 24 millas marinas (generalmente hasta 12 canceladas por el mar territorial) en la cual no cuenta con soberanía (como ya hemos señalado antes, ya que la única zona marítima que conforma propiamente «territorio» del Estado es el mar territorial). Sin embargo, para efectos de defender los objetos históricos y arqueológicos en la zona contigua, el Estado puede asumir que, si estos son retirados sin su permiso, se entenderán retirados de su mar territorial, con lo cual podrá iniciar una figura denominada el «derecho de persecución», que generalmente solo se mantiene hasta la zona contigua si se inicia propiamente dentro del mar territorial (ONU, 1982, art. 111).

Entonces, teniendo en consideración todo lo anterior, podemos concluir que las ficciones jurídicas son instituciones que se encuentran presentes en el derecho internacional y que no son ajenas al régimen especializado del derecho del mar. Asimismo, las ficciones jurídicas en el ordenamiento jurídico internacional pueden generar diversas ventajas en lo que a su aplicación respecta. El derecho del mar cuenta con ficciones jurídicas que pueden resultar beneficiosas para los Estados ribereños y la propuesta de una ficción jurídica por la cual se entienda que se congelan las líneas de base iría en la misma línea.

\subsection{La ficción jurídica presentada como cuestión a trabajar por parte de la CDI y los avances sobre el tema «La elevación del nivel del mar en relación con el derecho internacional»}

Este subpunto iniciará señalando brevemente la propuesta que ha sido presentada como la tercera cuestión a trabajar en relación al ámbito de la condición de Estado del tema «La elevación del nivel del mar en relación con el derecho internacional» en el programa de trabajo de la CDI en el informe de su septuagésima sesión. A partir de ello, dado que aún no se cuenta con acceso al avance de la CDI en relación a la cuestión señalada, se presentará información relevante del único avance que se ha dado hasta la fecha, el cual es el primer documento temático elaborado por los miembros Aurescu y Oral sobre las cuestiones relativas a otro ámbito de estudio sobre el tema: el del derecho del mar.

Como ya comentamos líneas arriba, la CDI aprobó la inclusión de una cuestión dentro del ámbito del tema ya previamente señalado que se lee a la letra como sigue:

Análisis de la ficción jurídica según la cual, considerando la «congelación» de las líneas de base y el respeto de las fronteras establecidas por tratados, sentencias judiciales o 
laudos arbitrales, podría admitirse la continuidad de la condición de Estado en el caso de los Estados insulares en razón de la presencia de territorios marítimos establecidos por la existencia previa de territorios bajo su soberanía antes de que estos quedaran completamente cubiertos por el mar o resultaran inhabitables. (CDI, 2019a, p. 360)

La septuagésima segunda sesión de la CDI originalmente se celebraría en el año 2020 sin interrupciones, pero fue pospuesta debido a la pandemia del Covid-19 sin señalar nueva fecha (Presidente de la Asamblea General de la ONU, 2020). Posteriormente, la Asamblea General dispuso que la septuagésima segunda sesión se llevaría a cabo del 26 de abril al 4 de junio y del 5 de julio al 6 de agosto del presente año (2020, párrafjo 17). Sin embargo, incluso si no se hubiera dado dicho suceso, originalmente se había previsto que la CDI presentaría su informe en relación con el ámbito de la condición de Estado para el año 2021, por lo que, de cualquier manera, aún no contaríamos con los avances de la CDI respecto a las cuestiones de este ámbito. Sin embargo, como ya se comentó, sí hubo un primer documento temático presentado respecto al ámbito del derecho del mar.

Al respecto, con la inclusión del tema ya mencionado a su agenda, la CDI aprobó la creación de un Grupo de Estudio a ser copresidido de manera rotativa con el fin de responder a la necesidad de flexibilidad y a la complejidad y variedad del tema (Galvão Teles, 2020, pp. 152-153). Precisamente, se aprobó que se trabajen tres ámbitos distintos: el derecho del mar, la condición de Estado y la protección de las personas afectadas por la elevación del nivel del mar (CDI, 2019a, pp. 360-361). Se programó que la primera cuestión sea presentada originalmente este año, por lo que, antes de que la Organización Mundial de la Salud declarara la existencia de una pandemia, se había preparado el avance que se mencionó, por lo que tenemos acceso a determinadas apreciaciones preliminares con respecto al ámbito del derecho del mar.

Una de las cuestiones a estudiar por parte de la CDI sobre el derecho del mar fue la de los «Posibles efectos jurídicos de la elevación del nivel del mar en las líneas de base y los límites exteriores de los espacios marítimos que se miden a partir de las líneas de base» (CDI, 2019a, p. 360). Al revisar su fraseo, comprendemos rápidamente que comparte la preocupación de la cuestión ya mencionada en materia de la condición de Estado, solo que no necesariamente en relación a la pérdida de esta última, sino de manera un tanto más general. Sin embargo, a nuestro parecer, existiría un punto de encuentro entre ambas cuestiones, que es en relación a la posibilidad de aplicar la ficción jurídica de congelación de las líneas de base.

La CDI ha señalado en su primer documento temático con respecto a la cuestión señalada que el Comité sobre las Líneas de Base de la Asociación de Derecho Internacional, institución compuesta por especialistas en derecho internacional de todo el mundo que guarda una estrecha cooperación con la CDI, adoptó un informe 
final en su conferencia desarrollada en Sofía, Bulgaria, en el año 2012, en el que concluyó que las líneas de base son «movibles» (lo que entendemos equiparable al concepto desarrollado de "ambulatorias») y que, por tanto, deben cambiar jurídicamente con las ampliaciones del mar hacia adentro de la línea de bajamar (Comité sobre las Líneas de Base de la Asociación de Derecho Internacional, citado por CDI, 2020, p. 33). De manera más preocupante aún y, con relación en cuanto a la ficción jurídica analizada, mencionó lo siguiente:

[...] el derecho vigente de la línea de base normal es de aplicación en los casos de alteración significativa de las costas debido a ganancias y pérdidas territoriales. Los Estados ribereños pueden proteger y preservar su territorio con refuerzos físicos, pero no mediante la ficción jurídica de una línea trazada que no representa la línea de la marea baja real. (Comité sobre las Líneas de Base de la Asociación de Derecho Internacional, citado por CDI, 2020, p. 33)

Analizando esto, podemos ver que la Asociación de Derecho Internacional apostaría por entender que la fórmula para proteger los territorios de pérdidas debidas al hundimiento por el aumento en el nivel del mar sería a través de la utilización de refuerzos físicos, como podrían ser, por ejemplo, los diques marinos de los Países Bajos para proteger la tierra de la erosión hídrica al tratarse de un Estado bajo el nivel del mar (Yamamoto y Esteban, 2014, p. 151). Sin embargo, no sería posible la aplicación de una ficción jurídica como la propuesta, básicamente por esta ser inexistente aún en el derecho internacional.

Siguiendo entonces lo dicho por el Comité sobre las Líneas de Base de la Asociación de Derecho Internacional, no existiría jurídicamente la posibilidad de aplicar la ficción jurídica de congelación de las líneas de base, al haber mencionado la imposibilidad de aplicar una "ficción jurídica de una línea trazada que no representa la línea de la marea baja real» en sus términos. Sin embargo, este informe no fue bien visto en tanto no ofreció soluciones reales a los problemas de múltiples Estados ribereños debidos al aumento en el nivel del mar, motivo por el cual, en la conferencia en Sidney del año 2018, se aprobó la resolución 5/2008 que contiene una propuesta sobre este tema a partir de una consulta del Comité sobre el Derecho Internacional y la Elevación del Nivel del Mar. La respuesta fue completamente distinta a la esbozada seis años antes, y señaló a la letra lo siguiente:

[...] por motivos de seguridad y estabilidad jurídicas, siempre que las líneas de base y los límites exteriores de las zonas marítimas de un Estado ribereńo o archipelágico hayan sido debidamente determinados de conformidad con la Convención sobre el Derecho del Mar de 1982, no puede exigirse una nueva modificación de esas líneas de base y esos límites en caso de que la variación del nivel del mar afecte a la realidad geográfica del litoral. (Comité sobre las Líneas de Base de la Asociación de Derecho Internacional, citado por CDI, 2020, p. 33) 
Entonces, en esta nueva respuesta, la Asociación de Derecho Internacional se encontraría proponiendo que, si se cumplen dos requisitos, los cuales son que medien motivos de seguridad y estabilidad jurídicas, y que se hayan respetado los criterios para el trazado de líneas de base previstos en la CONVEMAR, no se deba exigir a los Estados ribereńos que cumplan con su obligación jurídica de modificar las líneas de base tras el cambio físico en sus costas producto del aumento en el nivel del mar.

Siendo esto así, podemos notar que la propia Asociación de Derecho Internacional cambió de manera radical su parecer respecto a la posibilidad de mantener las líneas de base, así como también los límites marítimos a partir de dichas líneas, siempre que se cumplan los requisitos previamente señalados. Siendo así, si bien no se menciona de manera expresa, entendemos que la Asociación de Derecho Internacional ha pretendido señalar que sería posible aplicar una ficción jurídica de congelación de las líneas de base si se cumplen las condiciones indicadas.

Teniendo en cuenta todo lo señalado, podemos ver que algunos miembros de la CDI ya habían tomado en consideración la propuesta de la Asociación de Derecho Internacional relativa a la posibilidad de aplicar una ficción jurídica sobre la congelación de las líneas de base para efectos del ámbito del derecho del mar. Asimismo, luego de la revisión de este punto en el primer documento temático, el Grupo de Estudio concluyó que debería agregar mayor detalle sobre el trabajo de la Asociación de Derecho Internacional en su búsqueda de la lege ferenda para hacer frente a la elevación del nivel del mar y usarlo de insumo para su propio análisis (CDI, 2021, párr. 269). Con ello podemos notar que la CDI encuentra positivo el análisis realizado por la Asociación de Derecho Internacional sobre la posibilidad de aplicar la ficción jurídica de congelación de las líneas de base.

\subsection{Beneficios de la aplicación de la ficción jurídica para los Estados ribereños}

Finalmente, en este subpunto se presenta la posibilidad de aplicar la ficción jurídica planteada no solo como una excepción a fin de evitar la extinción de Estados específicos que se encuentren en riesgo sino también como una regla general para los Estados ribereños. Entre las ventajas se encontrarían una mayor estabilidad y seguridad jurídica cartográfica, lo cual implicará un menor número de controversias marítimas, y la posibilidad de mantener los límites exteriores de las zonas marítimas en los casos en los cuales estos se encuentran retrayéndose debido al aumento en el nivel del mar. Por otro lado, explicaremos cómo se pueden superar algunas preocupaciones inherentes a la aplicación de la ficción jurídica propuesta desde el propio derecho internacional. 
Como ya interpretamos del subpunto anterior, la Asociación de Derecho Internacional estableció que sería posible aplicar la ficción jurídica de congelación de las líneas de base siempre que esto tenga sustento en la seguridad o estabilidad jurídica. Adicionalmente, resulta importante precisar que lo expresado por dicha entidad no se trata de un simple proyecto académico sin respuesta positiva por parte de los Estados, ya que la CDI señala que se ha encontrado coincidencia por parte de muchos Estados en relación a ello (CDI, 2020, p. 33). Si bien esto no convierte a la ficción jurídica automáticamente en una norma internacional, consideramos que es un buen primer paso para su posible aplicación efectiva. Mencionamos esto ya que, para que la ficción jurídica planteada pueda aplicarse, debe encontrarse prevista en una norma jurídica internacional. Hacia el final de este subpunto, daremos una breve opinión sobre cuál sería la mejor fuente del derecho internacional para contener la posibilidad de aplicar la ficción jurídica propuesta como una norma jurídica.

Teniendo en cuenta lo mencionado por la Asociación de Derecho Internacional, la aplicación de una ficción jurídica de congelación de las líneas de base tendría como una ventaja que generaría evidentemente mayor seguridad jurídica en el ordenamiento jurídico internacional, con lo cual se generaría una satisfacción mutua entre la ficción y la seguridad jurídica requerida para poder aplicarla en los términos de la Asociación de Derecho Internacional. Al respecto, Anderson comenta que existen determinados problemas de falta de seguridad jurídica en el derecho del mar, los cuales son generados por los cambios tanto naturales como jurídicos de las líneas de base debidos a los motivos ya señalados (entre ellos, el aumento en el nivel del mar); a ello añade que incluso esto llega a afectar a los límites marítimos de manera derivada de estos cambios, especificando que algunos límites son ambulatorios y otros no (2008, p. 382). Asimismo, menciona que otro problema que genera falta de seguridad jurídica es los cambios en los mapas y cartografías oficiales (2008, p. 383), los cuales, como se vio líneas arriba, deben ser modificados constantemente conforme a los cambios en la geografía de los Estados ribereños, lo cual, en el caso de las líneas de base, implica no solo un cambio físico sino también jurídico.

Por otro lado, Rothwell y Stephens, identifican que el aumento en el nivel del mar debido al cambio climático generaría, teniendo en cuenta la regulación del derecho del mar actual, que se tengan que las líneas de base se tengan que "recalibrar» (to be recalibrated), lo que producirá definitivamente problemas en la seguridad jurídica internacional; entre los problemas, identifican incertidumbre en los límites exteriores que puede acrecentar las controversias de jurisdicción marítima, como la existente en la zona del mar de China Meridional; podría generar riesgos para la navegación debido al derretimiento de hielo y a la generación de huracanes y ciclones, lo cual además incidiría en los marinos que busquen salvataje; y ajustes respecto a las pobla- 
ciones pesqueras de especies marinas calificadas como especies transzonales y altamente migratorias (2010, p. 26). Todo esto podría evitarse de aplicarse la ficción jurídica propuesta.

Siendo así, podemos notar que la aplicación de la ficción jurídica de congelación de las líneas de base podría ayudar a que haya una mayor seguridad jurídica marítima. Esto tendría además sustento en que los límites exteriores de las zonas marítimas de los Estados ribereños se encontrarían inequívocamente definidos, tal como sucede con los pertenecientes a las plataformas continentales de los Estados partes de la CONVEMAR que han decidido beneficiarse de la ficción jurídica de congelación del artículo 76.9. De hecho, teniendo en cuenta la ya previa existencia de algunas plataformas continentales fijas en virtud de dicho artículo, sería inconsistente que el resto de zonas marítimas de los Estados beneficiados sí mantengan aún el carácter ambulatorio. A nuestro parecer, esta situación de desigualdad entre zonas marítimas de un mismo Estado generaría incertidumbre jurídica, ya que existirán espacios marítimos en los cuales se encuentre una zona fija que no coincidirá necesariamente con las líneas de base y las demás zonas marítimas. Sin perjuicio de ello, somos conscientes de que algunos miembros de la CDI no se encuentran convencidos de que se genere esta disparidad necesariamente al entender que, en realidad, la plataforma continental no permanece estática (CDI, 2021, párr. 279-280). No obstante, por lo explicado anteriormente, consideramos que la interpretación correcta (y compartida también por otros miembros de la CDI) es aquella que implica que sí podría haber disparidad. De ser así, el congelar las líneas de base y, por tanto, el resto de zonas marítimas, sería una solución.

De manera específica para los Estados que se encuentran actualmente en riesgo de perder su territorio y, por tanto, la condición de tales, encontraremos un beneficio adicional al del resto de Estados ribereños: la posibilidad de mantener su territorio y, con ello, la condición de Estados con la que siempre contaron. Esto se explicaría en tanto que, si el Estado ribereño congela sus líneas de base jurídicamente hablando (en tanto que fácticamente hablando los cambios geográficos en las marcas de agua que componen las líneas de base no dejarán de darse), las zonas marítimas con las que el Estado cuente, se mantendrán en tanto seguirán contando desde dónde proyectarse. Una de estas zonas marítimas sería jurídicamente aún parte del territorio del Estado: el mar territorial.

Si bien es cierto que, como identifica Aznar Gómez, en algún momento, se terminará por sumergir el espacio terrestre del Estado de manera inevitable y que, desde entonces, se generaría una contradicción con el ya mencionado principio «la tierra domina el mar», lo cierto es que la ficción jurídica representaría la lex especialis, por lo que lo que aplicaría sería una fórmula de excepción, a lo que el mismo autor deno- 
mina un nuevo uti possidetis iuris náutico (2013, pp. 43 y 49). Es también importante precisar que, si bien la CDI ha considerado necesario analizar a mayor profundidad el principio «la tierra domina el mar» (2021, párr. 278 y 294.b), no encontramos constancia de que se trate también de una norma imperativa del derecho internacional, por lo que no consideramos que sea imposible que cuente con excepciones.

Pero por otro lado, yendo más allá de la formalidad de considerar la aún existencia del mar territorial a pesar de la inexistencia fáctica de espacio terrestre, Rayfuse señala, a nuestro parecer acertadamente, que, en caso de haberse congelado las líneas de base, lo que se encuentre hacia adentro de las mismas, por más que en la práctica no sea tierra, procederá a calificarse jurídicamente como «aguas interiores», que es la calificación que el derecho del mar otorga a espacios marítimos que se encuentran hacia adentro de las líneas de base, las cuales son equivalentes a la tierra para efectos jurídicos (2013, p. 188). En ese sentido, también jurídicamente hablando, el espacio hacia adentro de las líneas de base congeladas, que será únicamente de agua tras el hundimiento definitivo de la tierra del Estado, calificará para efectos del derecho del mar como espacio terrestre, lo cual sirve también como argumento jurídico para alegar que no se incumpliría el principio «la tierra domina el mar», al menos desde una perspectiva netamente jurídica.

Viendo las posibles fórmulas de argumentación existentes para fundamentar que los Estados ribereños en riesgo no perderían su territorio de manera acorde con el derecho del mar actual, podemos concluir que sería posible mantener el territorio para estos Estados en caso de poder aplicar la ficción jurídica propuesta y, por tanto, aún contarían con dicho elemento de la definición de Estado, manteniendo así la condición de tales. Sin embargo, pueden surgir ciertas preocupaciones legítimas basadas en el derecho internacional al analizar la posibilidad de aplicar una ficción jurídica de este tipo. A continuación, analizaremos las que consideramos principales y les brindaremos respuesta.

El primer cuestionamiento que surgiría desde el punto de vista netamente jurídico sería que, para el derecho internacional público, la efectividad es un criterio de relevancia mayor que las ficciones al momento de establecer relaciones, derechos u obligaciones jurídicas. Al respecto, siguiendo la doctrina más clásica, comenta Pagliari, con relación a lo requerido para constituirse como Estado, que la efectividad sería el único requisito para la condición de Estado, concepto que refiere a lo real y verdadero, contrariamente a lo ilusorio o aparente; así, el requisito de efectividad haría necesario que exista una situación real para atribuirle consecuencias jurídicas (citado por López Martín, 2018, pp. 87-88). En ese sentido, siguiendo este concepto, no sería posible mantener la condición de Estado con la sola aplicación de una ficción 
jurídica, ya que, dentro de la ficción jurídica planteada, el Estado que mantiene su condición de tal lo hace en virtud de dicha ficción sin tener, en efecto, un territorio. Al no contar con la efectividad necesaria, entonces, siguiendo este criterio que, sin duda, encuentra mayor relevancia que las ficciones jurídicas dentro del derecho internacional en general, no habría ficción jurídica que pueda salvar la condición de Estado una vez que, en la realidad de los hechos, ya no exista el territorio.

Sobre el particular, López Martín comenta que, si bien es cierto que la efectividad es un criterio importante en relación con la existencia o no de un Estado, dado que no existe en el derecho internacional un órgano centralizado que pueda decidir objetivamente que un Estado existe o no, la práctica va a generar que sean los demás Estados quienes decidan si un ente califica o no como Estado a partir del reconocimiento del mismo (2018, p. 89). Siendo así, es posible jurídicamente que la comunidad internacional considere que un Estado cuenta con la condición de tal independientemente de si efectivamente reúne los elementos constitutivos necesarios. A esto podemos ańadir que, como previamente se señaló, la comunidad internacional permite mantener la condición de Estado en casos en que efectivamente no existe un gobierno a través de la ficción jurídica en la que se basa el principio de continuidad del Estado. Por otro lado, desde el punto de vista inverso, Virally señala que la efectividad, por sí sola, no basta para que un Estado, en la práctica, exista, ya que el simple hecho de que un ente reúna los elementos constitutivos necesarios para ello no genera que sea considerado un Estado instantáneamente, siendo que, más bien, se trata de un paso dentro de un proceso mayor (citado por López Martín, 2018, p. 90).

Otro cuestionamiento podría ser que la aplicación de esta ficción jurídica podría generar un impacto socioeconómico negativo debido a que, si bien el Estado ribereño mantendría formalmente su territorio, fácticamente sería imposible para su población permanecer en el agua, por lo que se generaría necesariamente un fenómeno de migración hacia otros Estados de la misma. Al respecto, como se mencionó en un inicio, la CDI se encuentra también estudiando el ámbito de «la protección de las personas afectadas por la elevación del nivel del mar», entre las cuales son relevantes dos cuestiones: «Determinación de la existencia de principios jurídicos internacionales aplicables a la evacuación, la reubicación y la emigración de personas al extranjero a causa de los efectos adversos de la elevación del nivel del mar» y «Posibles principios aplicables a la protección de los derechos humanos de los desplazados internos o las personas que migran debido a los efectos adversos de la elevación del nivel del mar» (CDI, 2018a, p. 361). Sin embargo, al igual que sucede con el ámbito de la condición de Estado, la CDI recién tenía programado originalmente dar avances sobre estas cuestiones para el año 2021. 
En su lugar, podemos identificar algunos alcances doctrinarios que han realizado propuestas prácticas a fin de evitar que se configure un efecto socioeconómico negativo, tanto en el Estado que mantendrá jurídicamente su territorio a pesar del hundimiento, como en el receptor de las migraciones del primero. Entre ellas, la que mejor responde a este primer cuestionamiento es, a nuestro parecer, la planteada por Burkett, que seńala que, dado que el Estado ribereño mantendría sus zonas marítimas intactas y fijas, estas podrían explotarse a fin de generar rentas que financien la reubicación de la población (citada por Crawford y Rayfuse, 2012, p. 250). De esta manera, se podría utilizar el único recurso que mantendría formalmente el Estado ribereño bajo su jurisdicción a fin de que el Estado receptor de las migraciones pueda acoger a su población sin problemas de un costo económico muy alto y, de hecho, podría beneficiarse económicamente de las zonas marítimas del Estado ribereño, las cuales, tratándose de islas, suelen ser muy extensas.

Por otro lado, se podría cuestionar que el aplicar una ficción jurídica de este tipo podría llevar a un impacto jurídico negativo en relación a los demás Estados ribereños con los que se comparta algún límite marítimo generando así alguna situación no equitativa. La preocupación de que una institución termine beneficiando más a unos Estados que a otros es legítima; sin embargo, a esto Caron ya había hecho la precisión de que, en realidad, no se generaría ningún beneficio inequitativo per se, dado que los Estados ribereños no estaría reivindicando algo adicional a lo que ya tenían (2009, pp. 16-17), es decir, solo se estarían valiendo de los derechos que ya habían adquirido hasta el momento en que se entienda que sus líneas de base son jurídicamente congeladas. Sin embargo, advierte de igual manera el autor que, ya por un tema de practicidad, sí podría generarse un resultado inequitativo como resultado del movimiento de determinados recursos marinos vivos, tales como las ya seńaladas especies transzonales y altamente migratorias (Caron, 2009, p. 17), en tanto por su movimiento natural, podrían terminar trasladándose definitivamente fuera de las zonas marítimas que habrían quedado congeladas por parte de determinados Estados ribereños.

No obstante, con respecto a esto último, cabe señalar que dicho movimiento se daría de manera independiente a la congelación de las líneas de base y que finalmente dependería del azar qué especies se mantendrían dentro de las zonas marítimas de un determinado Estado, sean estas ambulatorias o no. Además, hay que tener en cuenta que, de ser aplicable una ficción jurídica de este tipo, los eventuales beneficiarios serían los Estados ribereños en su totalidad, por lo que lo más lógico sería que todos ellos apliquen la ficción jurídica en su beneficio, con lo cual la congelación se daría de manera consensuada por las partes de un mismo límite marítimo, ya que no tendría sentido que algún Estado ribereño no se quisiera beneficiar evitando que el hundimiento le merme zonas marítimas. 
Otro cuestionamiento posible se daría en relación a los efectos de la aplicación de una ficción jurídica como la propuesta en relación con determinadas normas consuetudinarias generales que a su vez son principios que han sido utilizadas de manera general por los Estados ribereños para delimitar sus zonas marítimas. En particular, los Estados suelen delimitar sus zonas marítimas a partir de una norma general concreta que es el principio de equidistancia. La aplicación del principio de equidistancia para la delimitación marítima implica que se trace una línea equidistante cuando se encuentran las costas de dos Estados ribereños en situación de superposición, sean de forma adyacente o vis a vis. Así, la CONVEMAR establece en su artículo 15 que, de existir costas adyacentes o frente a frente, los Estados ribereńos tendrán derecho de trazar «una línea media cuyos puntos sean equidistantes de los puntos más próximos de las líneas de base a partir de las cuales se mida la anchura del mar territorial de cada uno de esos Estados». Ocurre lo propio en aplicación del mencionado principio para el resto de zonas marítimas.

Sobre la aplicación de este principio, la División de Asuntos Oceánicos y del Derecho del Mar Oficina de Asuntos Jurídicos de la ONU menciona que los Estados ribereños pueden realizarla hasta en tres formas distintas: una línea de estricta equidistancia, es decir, aquella que toma en cuenta todos los puntos de base permitidos por el derecho del mar; una línea equidistante simplificada, que solo toma en cuenta los puntos relevantes; y una línea equidistante ajustada o modificada, que es aquella en que los Estados acuerdan dar cierta flexibilidad a la línea generalmente por razones de equidad (2001, pp. 51-53). Es importante no confundir el concepto de equidistancia, que como hemos visto implica el trazado de una línea que se genere como resultado del punto medio de los puntos de base de las costas de los Estados, con el último señalado de equidad, que simplemente implica otorgar indiscriminadamente una cantidad igual de zonas marítimas a los Estados con zonas marítimas contrapuestas, independientemente de la proporción entre sus costas.

Consideramos que, si bien probablemente los límites marítimos habrán sido generados en virtud de este principio, en realidad no se estaría contraviniendo de ninguna manera al aplicar la ficción jurídica de congelación de las líneas de base. Esto se debería a dos motivos principalmente. En primer lugar, si bien el principio de equidistancia puede ser de utilidad para los Estados, así como también para tribunales internacionales llamados a realizar delimitaciones marítimas, al momento de delimitar las zonas marítimas contrapuestas, no es una obligación jurídica de los Estados ribereños el hacer uso de este. Si así lo desearan, por ejemplo, podrían delimitar sus zonas marítimas en virtud de la equidad, generando un resultado no equitativo por acuerdo mutuo. En segundo lugar, si se congelan las líneas de base en relación con Estados ribereños que sí establecieron sus límites en virtud del principio de equidis- 
tancia, la imagen que se obtendría sería la misma que hasta inmediatamente antes de la congelación, dado que la ficción jurídica propuesta no implica una adaptación de las líneas de base ni de los límites marítimos, sino todo lo contrario: plantea el mantenimiento de los límites marítimos hasta el punto en que se encuentren como una consecuencia lógica de la fijación jurídica de las líneas de base. Por último, de manera adicional a lo mencionado, no existiría a nuestro parecer un motivo por el cual no pudieran crearse nuevas normas jurídicas de carácter consuetudinario o que constituyan principios contrarios al principio de equidistancia como producto de la aplicación de la ficción jurídica propuesta, en tanto no cuenta con carácter imperativo.

Finalmente, tal como mencionamos al inicio de este subpunto, la posibilidad de aplicar una ficción jurídica en el derecho internacional se encuentra condicionada a que la misma se encuentre contenida en una norma jurídica internacional. En ese sentido, si bien no es materia de análisis a profundidad en el presente artículo, consideramos importante dar el alcance de cuál sería, a nuestro parecer, la fuente del derecho internacional que podría contener esta norma jurídica de manera más efectiva. Al respecto, Hayashi, plantea que una posibilidad sería adoptar una enmienda a la CONVEMAR que permita a los Estados ribereños valerse de la ficción jurídica propuesta (citado por Yamamoto y Esteban, 2014, p. 143). Sin embargo, consideramos que esta salida sería complicada dado que el proceso de celebración de un tratado multilateral puede ser muy tedioso (la propia Tercera Conferencia de la ONU sobre el Derecho del Mar que dio como resultado la adopción de la CONVEMAR demoró casi 10 años). Además, no todos los Estados ribereños del mundo son partes de la CONVEMAR (nuestro Estado es un caso emblemático de esto), por lo que no sería efectivo para todos en la fórmula propuesta por Hayashi.

En tal sentido, consideramos que la salida más fácil sería que los Estados mismos inicien una práctica por la cual declaren conjuntamente que congelan sus líneas de base en virtud de una conciencia jurídica de la existencia de dicha posibilidad en el derecho internacional. Sumando ambos elementos, los cuales constituirían una práctica reiterada y una conciencia de obligatoriedad, tendremos como resultado la creación de una nueva costumbre internacional que contenga la ficción jurídica propuesta. Consideramos que esta sería la opción más viable dado que no implicaría un proceso extenso de celebración como sí implicaría la adopción de un nuevo tratado, y, además, por todo lo ya seńalado, existen motivos importantes para que la mayoría de los Estados ribereños en el mundo, si es que no son todos, deseen participar de la creación de una norma jurídica que les permita valerse de una ficción jurídica que los beneficie. Finalmente, si bien es cierto que no todos los Estados del mundo son ribereños, no se requiere que literalmente todos los Estados que 
conforman la comunidad internacional participen de la creación de una costumbre internacional para que alcance carácter universal y, por último, nada obstaría a que puedan crearse costumbres conteniendo la ficción jurídica propuesta de carácter regional o local (Yamamoto y Esteban, 2014, pp. 147-148). Debemos hacer la precisión de que la CDI no ha podido encontrar, a la fecha, una práctica estatal suficiente que evidencie que los Estados ribereños entiendan fijas sus líneas de base (2021, párr. 260 y 268), por lo que es importante dejar en claro que la ficción jurídica constituye solo lege ferenda y no lege lata.

En conclusión, siguiendo todo lo señalando, podemos concluir que la posible aplicación de la ficción jurídica de congelación de las líneas de base podría generar beneficios considerables para los Estados ribereños, sobre todo desde el plano de la seguridad jurídica. Evidentemente esto alcanzaría con especial interés a los Estados ribereños que se encuentran en riesgo de desaparecer debido al aumento en el nivel del mar, pero no de manera exclusiva, ya que todos los Estados ribereńos en cierto sentido se podrían beneficiar. Esto podría generar que eventualmente la mayoría de Estados ribereños cooperen para convertir esta ficción propuesta, aún doctrinaria, en una norma jurídica internacional, probablemente de carácter consuetudinario para una mayor efectividad.

\section{Conclusiones}

Del presente trabajo, se pueden extraer las siguientes conclusiones:

En primer lugar, la elevación del nivel del mar como resultado del cambio climático es un hecho que va en aumento y que aqueja a los Estados ribereńos en su conjunto, con especial énfasis en los Estados ribereños en riesgo de ver sumergido completamente su espacio terrestre a causa de dicho problema. En relación con estos últimos, de ver sumergido su espacio terrestre de forma permanente, perderían el elemento territorio de la definición de Estado y, por consiguiente, perderían la condición de tales bajo el derecho internacional actual.

En segundo lugar, es importante encontrar soluciones prácticas a la problemática identificada a fin de mantener la seguridad jurídica internacional. Una de las propuestas más importantes, que inclusive ha sido planteada como cuestión por la CDI es la posibilidad de aplicar una ficción jurídica por la cual se entienda que las líneas de base de los Estados ribereños se congelarían, con lo cual, no habría mayor cambio jurídico a partir de los cambios geográficos en las costas. Para el caso de los Estados en riesgo de desaparecer como consecuencia del cambio climático, esto implicaría que mantengan su territorio jurídicamente hablando (por más que no 
cumplan con el criterio de efectividad al no contar con un espacio terrestre en los hechos) y, por tanto, podrían mantener la condición de Estado.

Finalmente, la ficción jurídica propuesta generaría una mayor seguridad jurídica y no generaría efectos negativos ante posibles cuestionamientos. Además, para poder ser aplicable, tendría que encontrarse contenida en una norma jurídica internacional. Sobre esto último, la fuente del derecho internacional más práctica para materializar la norma sería una costumbre internacional universal, la cual sería posible de lograr con la intervención de los Estados ribereños interesados y los demás Estados del mundo, ya que se beneficiarían de todas maneras.

\section{Referencias bibliográficas}

Anderson, D. (2008). Modern Law of the Sea Selected Essays. Martinus Nihjoff. Colección Publications on Ocean Development [Vol. 59].

Asamblea General de la Organización de las Naciones Unidas (2020). Informe de la Comisión de Derecho Internacional sobre la labor realizada en su $72^{\circ}$ periodo de sesiones (A/74/10). Versión en español. https://documents-dds-ny.un.org/doc/UNDOC/GEN/ N20/368/26/PDF/N2036826.pdf?OpenElement

Aust, A. (2005). Handbook of International Law. Cambridge University Press. https://doi. org/10.1017/CBO9780511494123

Aznar Gómez, M. J. (2013). El Estado sin territorio: La desaparición del territorio debido al cambio climático. Revista electrónica de estudios internacionales (REEI), (26), 23-45. http://www.reei.org/index.php/revista/num26/articulos/ estado-sin-territorio-desaparicion-territorio-debido-al-cambio-climatico

Blanchard, C. (2015). Evolution or Revolution? Evaluating the Territorial State-Based Regime of International Law in the Context of the Physical Disappearance of Territory Due to Climate Change and Sea-Level Rise. The Canadian Yearbook of International Law, 53(53), 66-118. https://doi.org/10.1017/cyl.2016.4

Caron, D. (1990). When law makes climate change worse: rethinking the law of baselines in light of a rising sea level. Ecology Law Quarterly, 17(4), 621-653. https://www.researchgate.net/publication/254557086_When_Law_Makes_Climate_Change_Worse_ Rethinking_the_Law_of_Baselines_in_Light_of_a_Rising_Sea_Level

Caron, D. (2009). Climate change, sea level rise and the coming uncertainty in oceanic boundaries: a proposal to avoid conflict. En J. M. Van Dyke, y Seoung-Yong H. (Eds.), Maritime Boundary Disputes, Settlement Processes, and the Law of the Sea (pp. 1-18). Martinus Nijhoff.

Cazenave, A. y Remy, F. (2011). Sea level and climate: measurements and causes of changes. Wiley Interdisciplinary Reviews: Climate Change, 2(5), 647-662. https://doi. org/10.1002/wcc.139

Churchill, L. L. y Lowe, A. V. (1988). The Law of the Sea. 2a ed. Manchester University Press. 
Comisión de Derecho Internacional de la Organización de las Naciones Unidas [CDI]. (2006). Informe del Grupo de Estudio de la Comisión de Derecho Internacional "fragmentación del derecho internacional: dificultades derivadas de la diversificación y expansión del derecho internacional», elaborado por Martti Koskenniem (A/CN.4/L.682). Versión en espańol. https://documents-dds-ny.un.org/doc/UNDOC/LTD/G06/610/80/ PDF/G0661080.pdf?OpenElement

Comisión de Derecho Internacional de la Organización de las Naciones Unidas [CDI]. (2018a). Informe de la Comisión de Derecho Internacional (70º periodo de sesiones) (A/73/10). Versión en español. https://documents-dds-ny.un.org/doc/UNDOC/ GEN/G18/252/70/PDF/G1825270.pdf?OpenElement

Comisión de Derecho Internacional de la Organización de las Naciones Unidas [CDI]. (2018b). Informe de la Comisión de Derecho Internacional ( $70^{\circ}$ periodo de sesiones) - (A/73/10). Versión en inglés. https://documents-dds-ny.un.org/doc/UNDOC/ GEN/G18/252/67/PDF/G1825267.pdf?OpenElement

Comisión de Derecho Internacional de la Organización de las Naciones Unidas [CDI]. (2019a). Informe de la Comisión de Derecho Internacional ( $71^{\circ}$ periodo de sesiones) (A/74/10). Versión en español. https://documents-dds-ny.un.org/doc/UNDOC/ GEN/G19/243/96/PDF/G1924396.pdf?OpenElement

Comisión de Derecho Internacional de la Organización de las Naciones Unidas [CDI]. (2019b). Informe de la Comisión de Derecho Internacional ( $71^{\circ}$ periodo de sesiones) - (A/74/10). Versión en inglés. https://documents-dds-ny.un.org/doc/UNDOC/ GEN/G19/243/93/PDF/G1924393.pdf?OpenElement

Comisión de Derecho Internacional de la Organización de las Naciones Unidas [CDI]. (2020). Primer documento temático, preparado por Bogdan Aurescu y Nilüfer Oral, copresidentes del Grupo de Estudio sobre el tema "La elevación del nivel del mar en relación con el derecho internacional» (A/CN.4/740). Versión en español. Recuperado de https:// documents-dds-ny.un.org/doc/UNDOC/GEN/N20/053/94/PDF/N2005394. pdf?OpenElement

Comisión de Derecho Internacional de la Organización de las Naciones Unidas [CDI]. (2021). Avance del Informe de la Comisión de Derecho Internacional (72º periodo de sesiones) - (A/76/10). Versión en inglés. https://legal.un.org/ilc/reports/2021/ english/a_76_10_advance.pdf

Corte Internacional de Justicia [CIJ]. (1951). Sentencia de fondo del caso «Fisheries Csa (United Kingdom v. Norway). https://www.icj-cij.org/public/files/case-related/5/00519511218-JUD-01-00-EN.pdf

Crawford, E. y Rayfuse, R. (2012). Climate change and statehood. En R. Rayfuse, y S. V. Scott (Eds.), International Law in the Era of Climate Change (pp. 243-253). Edward Elgar Publishing.

Crawford, J. (2006). The creation of States in International Law. 2a ed. Oxford University Press. http://dx.doi.org/10.1093/law/9780199228423.001.0001

Crawford, J. (2012). Brownlie's Principles of Public International Law. 8a ed. Oxford, Oxford University Press. http://dx.doi.org/10.1093/he/9780199699698.001.0001 
Diez de Velasco, M. (2013). Instituciones del Derecho Internacional Público. 18a ed. Tecnos.

División de Asuntos Oceánicos y del Derecho del Mar Oficina de Asuntos Jurídicos de las Naciones Unidas (2001). Manual de delimitación de fronteras maritimas. Naciones Unidas. https:/www.un.org/Depts/los/doalos_publications/publicationstexts/ Handbook\%20on\%20the\%20delimitation\%20of\%20maritime\%20boundary_Spa. pdf

Dremliuga, R. (2017). A Note on the Application of Article 234 of the Law of the Sea Convention in Light of Climate Change: Views from Russia. Ocean Development \& International Law, 48(2), 1-8. http://dx.doi:10.1080/00908320.2017.1290486

Ezcurra, P. y Rivera-Collazo, I. (2018). An assessment of the impacts of climate change on Puerto Rico's Cultural Heritage with a case study on sea-level rise. Journal of Cultural Heritage, 32, 1-12. https://doi.org/10.1016/j.culher.2018.01.016

Fernández Huertas, J. M. (1984). Estudio jurídico de la frontera aérea del Estado (tesis de doctorado, Universidad Complutense de Madrid, España). https://eprints.ucm.es/id/ eprint/53286/1/5309873334.pdf

Foro de Islas del Pacífico con Misiones en las Naciones Unidas (2019). Submission of the Members of the Pacific Islands Forum to the International Law Commission on the Topic of Sea-Level Rise in Relation to International Law. Anexo de la nota diplomática S/N de 30 de diciembre de 2019 remitida al secretario de la Comisión de Derecho Internacional de la Organización de las Naciones Unidas. https://legal.un.org/ilc/ sessions/72/pdfs/english/slr_pif.pdf

Galvão Teles, P. (2020). Sea-Level Rise in Relation to International Law: A New Topic for the United Nations International Law Comission. En M. Chantal Ribeiro, F. Loureiro Bastos y T. Henriksen (Eds.), Global Challenges and the Law of the Sea. Springer Nature Switerzerland.

Garner, B. A. (Ed.). (2004). Black's Law Dictionary. 8a ed. West Group.

Grote Stoutenburg, J. (2013). When Do States Disappear? Thresholds of Effective Statehood and the Continued Recognition of «Deterritorialized» Island States. En M. B. Gerrard, y G. E. Wannier (Eds.), Threatened island nations: legal implications of rising seas and a changing climate (pp. 57-87). Cambridge University Press. https://doi.org/10.1017/ CBO9781139198776

Grote Stoutenburg, J. (2015). Disappearing Island States in International Law. Brill Nijhoff. http://dx.doi.org/10.1163/9789004303010

López Martín, A. G. (2018). Creación de Estados en el Derecho internacional contemporáneo en casos de secesión: efectividad /legalidad. Anuario Hispano-Luso-Americano de derecho internacional, (23), 75-122. https://ihladi.net/wp-content/uploads/2018/02/ Creacion-de-Estados-en-el-Derecho-internacional-contemporaneo-en-casos-desecesion.pdf

Milanovic, M. (2014) The Lost Origins of Lex Specialis Rethinking the Relationship between Human Rights and International Humanitarian Law. En J. D. Ohlin (Ed.), Theoretical Boundaries of Armed Conflict and Human Rights (pp. 78-117). Cambridge University Press. https://doi.org/10.1017/CBO9781316481103 
Misión Permanente de la República de Maldivas en las Naciones Unidas (2019). Information and examples of State practice on "Sea-level rise in relation to international law" A submission by the Republic of Maldives. Anexo de la nota diplomática $\mathrm{N}^{\circ}$ 2019/ UN/N/50 de 31 de diciembre de 2019 remitida a la Oficina de Asuntos Legales de la Organización de las Naciones Unidas. https://legal.un.org/ilc/sessions/72/pdfs/ english/slr_maldives.pdf

McAdam, J. (2010). 'Disappearing States', Statelessness and the Boundaries of International Law. En J. McAdam (Ed.), Climate Change and Displacement Multidisciplinary Perspectives (pp. 105-129). Hart Publishing.

Organización de las Naciones Unidas [ONU]. (1958). Convención sobre el Mar Territorial y la Zona Contigua. https://www.dipublico.org/10524/ convencion-sobre-el-mar-territorial-y-la-zona-contigua-ginebra-29-de-abril-de-1958/

Organización de las Naciones Unidas [ONU]. (1982). Convención de las Naciones Unidas sobre el Derecho del Mar [CONVEMAR]. Versión en español. https://www.un.org/ Depts/los/convention_agreements/texts/unclos/convemar_es.pdf

Park, S. (2011). El cambio climático y el riesgo de apatridia: La situación de los Estados insulares bajos. Alto Comisionado de las Naciones Unidas para los Refugiados (ACNUR). https://www.acnur.org/5d546a124.pdf

Powers, A. y Stucko, C. (2013). Introducing the Law of the Sea and the Legal Implications of Rising Sea Leveles. En M. B. Gerrard y G. E. Wannier (Eds.), Thereatened island nations: legal implications of rising seas and a changing climate. Cambridge University Press. https://doi.org/10.1017/CBO9781139198776

Presidente de la Asamblea General de la ONU(2020). Draft decision entitled «Postponement of the seventy-second session of the International Law Commission» (A/74/L.70). https:// undocs.org/es/A/74/L.70

Rayfuse, R. (2012). Climate change and the Law of the Sea. En R. Rayfuse, y S. V. Scott (Eds.), International Law in the Era of Climate Change (pp. 147-174). Edward Elgar Publishing.

Rayfuse, R. (2013). Sea Level Rise and Maritime Zones: Preserving the Maritime Entitlements of «Disappearing» States. En M. B. Gerrard, y G. E. Wannier (Eds.), Threatened island nations: legal implications of rising seas and a changing climate (pp. 167-191). Cambridge University Press. https://doi.org/10.1017/CBO9781139198776.

Remiro Brotóns, A., Riquelme Cortado, R., Orihuela Calatayud, E., Diez-Hochleitner, J., y Pérez-Prat Durbán, L. (2010). Derecho Internacional. Curso General. Tirant lo Blanch.

República de Maldivas (2008). Constitution of the Republic of Maldives 2008. Anexo de la nota diplomática N²019/UN/N/50 de 31 de diciembre de 2019 remitida a la Oficina de Asuntos Legales de la Organización de las Naciones Unidas. https://legal.un.org/ilc/ sessions/72/pdfs/english/slr_maldives.pdf

Rothwell, D. y Stephens, T. (2010). The International Law of the Sea. 2a ed. Hart Publishing.

Séptima Conferencia Internacional Americana. (1933). Convención sobre Derechos y Deberes de los Estados [Tratado de Montevideo]. https://www.dipublico.org/14602/ 
convencion-sobre-derechos-y-deberes-de-los-estados-septima-conferencia-internacional-americana-montevideo-1933/

Shaw, M. (2006). International Law. 6a ed. Cambridge University Press. https://doi. org/10.1017/CBO9781139051903

Siegel, F. R. (2020). Adaptations of Coastal Cities to Global Warming, Sea Level Rise, Climate Change and Endemic Hazards. Springer Nature Switzerland. https://doi. org/10.1007/978-3-030-22669-5

Symmons, C.R. y Reed, M. W. (2010). Baseline Publicity and Charting Requirements: An Overlooked Issue in the UN Convention on the Law of the Sea. Ocean Development \& International Law, (41) 77-111. https://doi.org/10.1080/00908320903510068

Sornarajah, M. (2010). The International Law on Foreign Investment. 3a ed. Cambridge University Press. https://doi.org/10.1017/CBO9780511841439

Strati, A. (2006). Protection of the underwater cultural heritage: From the shortcomings of the UN Convention on the Law of the Sea to the compromises of the UNESCO Convention. En A. Strati, M. Gavouneli y N. Skourtos (Eds.), Unresolved Issues and New Challenges to the Law of the Sea Time Before and Time After (pp. 21-62). Martinus Nihjoff. Colección Publications on Ocean Development [Vol. 54].

Tanaka, Y. (2019). The International Law of the Sea. 3a ed. Cambridge University Press. http://dx.doi.org/10.1017/9781108545907

Walker, G. (2012). Definitions for the Law of the Sea. Martinus Nijhoff.

Yamamoto, L. y Esteban, M. (2014). Atoll Island States and International Law: Climate Change Displacement and Sovereignty. Springer-Verlag Berlin Heidelberg. http:// dx.doi.org/10.1007/978-3-642-38186-7

Fecha de recepción: 16 de abril de 2021

Fecha de aprobación: 26 de agosto de 2021 